\title{
Invariants for Smooth Conjugacy of Hyperbolic Dynamical Systems. IV
}

\author{
R. de la Llave ${ }^{1, \star}$ and R. Moriyón ${ }^{2}$ \\ ${ }^{1}$ Department of Mathematics, Fine Hall, Washington Road, Princeton University, \\ Princeton, NJ 08544, USA \\ ${ }^{2}$ División de Matemáticas, Univ. Autónoma de Madrid, Cantoblanco, E-28049 Madrid, Spain
}

\begin{abstract}
We show that if two $C^{\infty}$ transitive Anosov flows in a threedimensional manifold are topologically conjugate and the Lyapunov exponents on corresponding periodic orbits agree, then the conjugating homeomorphism is $C^{\infty}$.
\end{abstract}

\section{Introduction and Statement of Results}

The purpose of this paper is to present a unified approach to the problem of smooth conjugacy of Anosov systems (both flows and diffeomorphisms) in low dimensional manifolds. Our results extend those of [Ll] for diffeomorphisms in that no proximity assumption is made. Also, the results for flows, [MM], are extended to cover arbitrary flows instead of one-parameter families of them. We also recover the result of [MM] that all Anosov diffeomorphisms of a twodimensional manifold with constant Lyapunov exponents in periodic orbits are $C^{\infty}$ conjugate to linear automorphisms. Finally, we show how the important result of Feldman and Ornstein, [FO], on $C^{1}$ conjugacy of geodesic flows implies $C^{\infty}$ conjugacy.

We have known from Palis that Ghys has suggested a different approach using one-dimensional expansive maps to prove $C^{1}$ regularity of the conjugacy.

Our results are the following:

Theorem 1. Let $X, Y$ be two $C^{\infty}$ transitive Anosov vector fields in a compact threedimensional manifold. If they are $C^{0}$ conjugated and the Lyapunov exponents at corresponding periodic orbits are the same, then the conjugating homeomorphism is $C^{\infty}$.

Remark. Notice that the hypothesis about the Lyapunov exponents follows from $C^{1}$, or even Lipschitz, conjugacy. Hence, $\mathrm{C}^{1}$ conjugacies are $\mathrm{C}^{\infty}$ for the systems considered in Theorem 1. This remark also applies to Theorem 2.

* Partially supported by NSF grant \# DMS 85-04984 
Remark. In the particular case of Anosov vector fields with $C^{1}$ stable and unstable foliations (which includes geodesic flows on two-dimensional manifolds with negative curvature) Feldman and Ornstein, [FO], have shown that all $C^{0}$ conjugacies are $C^{1}$. By the previous remark, they are $C^{\infty}$.

Our theorem has some more applications for geodesic flows on surfaces with a negatively curved metric.

Out of Morse-Gromov construction - which we learned from [Ka] - we can conclude that the space of negatively curved metrics on a fixed surface is connected and, moreover that the homotopy class of a geodesic determines it uniquely.

Then, by Anosov structural stability theorem, all geodesic flows of negatively curved metrics on the same surface are orbit equivalent and the equivalence has to preserve the homotopy class of geodesics.

It then follows that if the lengths of closed geodesics in corresponding homotopy classes are the same and if the Lyapunov exponents also agree, we have that the flows are $C^{\infty}$ conjugate.

These invariants come very close to be those that one can recover from the spectrum of the Laplacian. The Duistermaat-Guillemin trace formula [DG] whose assumptions are verified for negatively curved metrics - implies that one can recover the lengths and the Lyapunov exponents of closed geodesics from the spectrum of the Laplacian. Unfortunately, it does not seem to be known how to recover information about the homotopy classes of the geodesics from spectrum.

Notice that the Feldman-Ornstein theorem shows that the lengths of closed geodesics on each homotopy class determine the Lyapunov exponents. If the homotopy class could be found out from the spectrum of the Laplacian, then the geometric information provided by the spectrum would be very redundant!

Theorem 2. Let $f$ and $g$ be two $C^{\infty}$ Anosov diffeomorphisms in a compact twodimensional manifold. If they are $C^{0}$ conjugate and the Lyapunov exponents at corresponding periodic orbits are the same, then the conjugating homeomorphism is $C^{\infty}$.

Remark. Transitivity does not appear in the hypothesis of Theorem 2, because a theorem of Newhouse, $[\mathrm{Ne}]$, shows that all Anosov diffeomorphisms in twodimensional manifolds are transitive.

Indeed it is known that the only two-dimensional manifolds that admit Anosov diffeomorphisms are two-dimensional tori and that all the Anosov diffeomorphisms on them are topologically conjugate to linear automorphsims [Fr] (see also [Ma]). Hence, given two Anosov diffeomorphisms in a two-dimensional manifold, $-T^{2}$ - topological conjugacy is implied by having conjugate actions in the first cohomology group.

It would be interesting to extend the previous results to higher dimensions. Lopez de Sa has pointed out that diffeomorphisms of the type $f(x, y, u, v)=(2 x+y$, $x+y, 2 u+v, u+v), g(x, y, u, v)=f(x, y, u, v)+(0,0, \eta(x, y), p(x, y))$ can be considered having the same Lyapunov exponents at periodic orbits although the derivatives at fixed points are not linearly conjugate, so that the conjugacy cannot be even $C^{1}$.

Another interesting open question would be to extend the results here to analytic conjugacy. 


\section{Proofs of the Theorems}

Theorem 2 is a consequence of Theorem 1 by a standard suspension argument. It is easy to check that two topologically conjugate diffeomorphisms give rise to topologically equivalent manifolds and that the suspension flows are topologically conjugate on the resulting manifold.

The strategy of the proof of Theorem 1 is based on that of [Ll]: first we prove Lipschitz regularity of the conjugation using scattering theory, and then we "bootstrap" the regularity measured in the classes $C_{s}^{k}, C_{u}^{k}$ introduced in [LMM].

The key element that allows us to treat both flows and systems not necessarily close to each other is the construction of well behaved smooth coordinates in stable manifolds by regularizing carefully the initial conjugating homeomorphism. This global construction permits us to work in the manifold itself instead of using real coordinates, and the construction is more natural than that of [L1]. A global construction of this sort for an infinitesimal problem can be found in [MM].

Before giving the proof of Theorem 1 we recall some definitions from [LMM].

Given a $C^{0}$ foliation $\mathscr{F}$ of a manifold $M$ by $C^{\infty}$ leaves with continuous defining jets (that is, the leaves are locally a continuous family of $C^{\infty}$ imbedded disks), we say that a function defined on $M$ is of class $C_{\mathscr{F}}^{\infty}$ if it is of class $C^{\infty}$ on each leaf of $\mathscr{F}$, and the $C^{\infty}$-jet of that restriction depends continuously on the base point. We similarly define $C_{\mathscr{F}}^{\infty}$ maps between $M$ and another manifold $N$. In case $\mathscr{F}$ is the stable (respectively unstable) foliation associated to an Anosov system, we will denote $C_{\mathscr{F}}^{\infty}$ by $C_{s}^{\infty}$ (respectively $C_{u}^{\infty}$ ). When possibility of confusion arises because there are several vector fields, we will indicate the vector field in parenthesis, e.g. $C_{s(X)}^{\infty}$. We will also denote by $C_{X}^{\infty}$ the space of functions $C_{\mathscr{F}}^{\infty}$ with respect to integral curves of the vector field $X$.

Proof of Theorem 1. Let us denote by $\tau_{t}$ and $\sigma_{t}$ the flows of $X$ and $Y$ respectively. Finally, $\phi$ will be the homeomorphism that conjugates the flows, $\tau_{t}=\phi \circ \sigma_{t} \circ \phi^{-1}$. To show that $\phi$ is $C^{\infty}$, it clearly suffices to show that a cover is $C^{\infty}$. Hence we can assume without loss of generality that the stable foliations of $X$ and $Y$ admit tangent vector fields of unit length which we will denote by $X^{s}$ and $Y^{s}$ respectively.

An important ingredient in our argument is the following lemma that shows that $\phi$ can be approximated by more regular homeomorphisms.

Lemma. If $\mathscr{F}$ and $\mathscr{G}$ are $C^{0}$ foliations of the compact manifold $M$ with onedimensional $C^{\infty}$ leaves, then any homeomorphism $\phi$ of $M$ that sends leaves of $\mathscr{F}$ to leaves of $\mathscr{G}$ can be approximated uniformly by $C_{\mathscr{F}}^{\infty}$ homeomorphisms that send each leaf of $\mathscr{F}$ to its image under $\phi$, and whose inverses are of class $C_{\mathscr{G}}^{\infty}$.

The proof of the lemma will be postponed to the end. We apply it now to the conjugating homeomorphism $\phi$ and the foliations by stable manifolds of $X$ and $Y$ to produce another diffeomorphism $\tilde{\phi}$ in $C_{s(X)}^{\infty}$.

We will show that $\psi=\phi \circ \tilde{\phi}^{-1}$ is in $C_{s(X)}^{\infty}$. Then $\phi$ itself should be in $C_{s(Y)}^{\infty}$. A similar argument with $-X,-Y$, would show that $\phi$ is in $C_{u(Y)}^{\infty}$, and it is clear it is in $C_{Y}^{\infty}$. Then, an appeal to Lemma 2.3 of [LMM] (an alternative proof is [Jo]) will show $\phi \in C^{\infty}$ and establish the theorem.

Using the regularized homeomorphism $\tilde{\phi}$ we introduce another flow $\tilde{\tau}_{t}=\tilde{\phi} \sigma_{t} \tilde{\phi}^{-1}$ - which we can assume is $C^{0}$ close to $\tau_{t}$. We will show that, under the hypothesis of the theorem, any homeomorphism intertwining $\tau_{t}$ and $\tilde{\tau}_{t}$ is in $C_{s(X)}^{\infty}$. 
To show that $\psi \in C_{S(X)}^{\infty}$, we will prove that

$$
\psi=\lim _{t \rightarrow \infty} \tau_{t} \circ \tilde{\tau}_{-t}
$$

in the $C^{0}$ sense. This will enable us to conclude that $\psi$ is Lipschitz along stable directions of $X$ by estimating the Lipschitz constant of $\tau_{t} \circ \tilde{\tau}_{-t}$ along stable directions independently of $t$. From that point, on the bootstrap of regularity is much easier.

Since $\tau_{t} \tilde{\tau}_{-t}(x)=\tau_{t} \circ \psi^{-1} \circ \tau_{-t} \circ \psi(x)$, it suffices to show that $\tau_{t} \circ \psi^{-1} \circ \tau_{-t}$ converges uniformly to the identity. This follows, by a well known argument, from the fact that $\psi^{-1}$ preserves contractive leaves, that we can bound the distance along the leaf between $\psi^{-1} \circ \tau_{-1}(x)$ and $\tau_{-t}(x)$ independently of $x$ and $t$ and the uniform contractivity of $\tau_{t}$ along the stable leaves.

We start now to show that $\psi$ is uniformly Lipschitz along stable manifolds of $X$. It suffices to find a bound for the derivative of $\tau_{t} \circ \tau_{-t}$ along $X^{s}$ uniform in $t$ and $x$. Computing, we get

$$
\begin{aligned}
\left(\tau_{t} \circ \tilde{\tau}_{-t}\right)^{\prime}(x) \cdot X^{s}(x)=\left(\tau_{t} \circ \tilde{\phi} \circ \sigma_{-t} \circ \tilde{\phi}^{-1}\right)^{\prime}(x) \cdot X^{s}(x) & \\
= & \tilde{\xi}\left(\tilde{\phi}^{-1}(x)\right)^{-1} \cdot \Lambda_{t}^{(Y)}\left(\sigma_{-t} \circ \tilde{\phi}^{-1}(x)\right)^{-1} \cdot \tilde{\xi}\left(\tilde{\phi} \circ \sigma_{-t} \circ \tilde{\phi}^{-1}(x)\right) \cdot \Lambda_{t}^{(X)}\left(\tilde{\phi} \circ \sigma_{-t} \circ \tilde{\phi}^{-1}(x)\right) \\
= & {\left[\xi\left(\tilde{\phi} \circ \sigma_{-t} \circ \tilde{\phi}^{-1}(x)\right) / \tilde{\xi}\left(\tilde{\phi}^{-1}(x)\right)\right] \cdot\left[\Lambda_{t}^{(X)}\left(\phi \circ \sigma_{-t} \circ \tilde{\phi}^{-1}(x)\right) / \Lambda_{t}^{(Y)}\left(\sigma-t \circ \tilde{\phi}^{-1}(x)\right)\right] } \\
& \times\left[\Lambda_{t}^{(X)}\left(\tilde{\phi} \circ \sigma_{-t} \circ \tilde{\phi}^{-1}(x)\right) / \Lambda_{t}^{(X)}\left(\phi \circ \sigma_{-1} \circ \tilde{\phi}^{-1}(x)\right)\right],
\end{aligned}
$$

where we have used that

$$
\begin{gathered}
\tilde{\phi}^{\prime}(y) \cdot Y^{s}(y)=\tilde{\xi}(y) \cdot X^{s}(\tilde{\phi}(y)), \\
\sigma_{t}^{\prime}(x) \cdot Y^{s}(y)=\Lambda_{t}^{(Y)}(y) \cdot Y^{s}\left(\sigma_{t}(y)\right), \\
\tau_{t}^{\prime}(x) \cdot X^{s}(x)=\Lambda_{t}^{(X)}(x) \cdot X^{s}\left(\tau_{t}(x)\right)
\end{gathered}
$$

for certain positive functions $\tilde{\xi}, \Lambda_{t}^{(Y)}, \Lambda_{t}^{(X)}$ in $C_{s(Y)}^{\infty}$. The first follows from the fact that $\tilde{\phi}$ is $C_{s(Y)}^{\infty}$ and maps leaves of the stable foliation for $Y$ into leaves of the stable foliation of $X . A$ can be computed by

$$
\Lambda_{t}^{(X)}(x)=\exp \left[-\int_{0}^{t} \lambda_{X}^{s}\left(\tau_{r} x\right) d r\right]
$$

where $\left[X, X^{s}\right]=\lambda_{X}^{s} X^{s}$, and similarly for $\Lambda_{t}^{(Y)}(x)$.

We will prove that each term in the right-hand side of (1) is bounded by a constant independent of $t$ and $x$.

The first factor between brackets is bounded by a constant independent of $t$ and $x$ since $\xi$ is a nowhere vanishing continuous function. In order to prove that the last term is also bounded by a constant independent of $t$, we call $\delta=\max d(\phi(x), \widetilde{\phi}(x))$, where the distance is measured along the stable leaf containing both of them. We consider the points $x_{0}=\tilde{\phi} \circ \sigma_{-t} \circ \widetilde{\phi}^{-1}(x)$ and $x_{1}=\phi \circ \sigma_{-t} \circ \widetilde{\phi}^{-1}(x) \in W_{X}^{s}(x)$. The 
distance between $x_{0}$ and $x_{1}$ is smaller than $\delta$. Then, by (2),

$$
\begin{aligned}
\left|\Lambda_{t}^{(X)}\left(x_{0}\right)-\Lambda_{t}^{(X)}\left(x_{1}\right)\right| & =\left|\exp \left[-\int_{0}^{t} \lambda_{X}^{s}\left(\tau_{r}\left(x_{0}\right)\right) d r\right]-\exp \left[-\int_{0}^{t} \lambda_{X}^{s}\left(\tau_{r}\left(x_{1}\right)\right) d r\right]\right| \\
& \leqq \max \left(\left|\Lambda_{t}^{(X)}\left(x_{0}\right)\right|,\left|\Lambda_{t}^{(X)}\left(x_{1}\right)\right|\right) \int_{0}^{t}\left|\lambda_{X}^{s}\left(\tau_{r}\left(x_{0}\right)\right)-\lambda_{X}^{s}\left(\tau_{r}\left(x_{1}\right)\right)\right| d r \\
& \leqq C \max \left(\left|\Lambda_{t}^{(X)}\left(x_{0}\right)\right|,\left|\Lambda_{t}^{(X)}\left(x_{1}\right)\right|\right) \mid\left\|\lambda_{X}^{s}\right\|_{C^{\alpha}} \delta^{\alpha}
\end{aligned}
$$

where we have taken into account that $\lambda_{X}^{s}$ is of class $C^{\alpha}$ (which is a consequence of the $C^{1+\alpha}$ smoothness of the center stable bundle) and that $d\left(\tau_{r}\left(x_{0}\right), \tau_{r}\left(x_{1}\right)\right)$ $\leqq K \lambda^{r} d\left(x_{0}, x_{1}\right)$ for some $\lambda<1$; this exponential decay allows us to get a bound for the integral independent of $t$. If we divide both sides of the above inequality by $\left|\Lambda_{t}^{(X)}\left(x_{1}\right)\right|$,- which, clearly, is bounded away from zero - we get the desired estimate.

Finally, we show that $\left|\Lambda_{t}^{(X)}\left(\phi \circ \sigma_{-t} \circ \tilde{\phi}^{-1}(x)\right) / \Lambda_{t}^{(Y)}\left(\sigma_{-t} \circ \tilde{\phi}^{-1}(x)\right)\right|$ is bounded uniformly in $t$ and $x$. This is a consequence of the fact that corresponding periodic orbits $X$ and $Y$ have the same Lyapunov exponents. Given a periodic orbit $\gamma$ of $Y$ of period $T$, its stable Lyapunov exponent by (2) is

$$
L_{s}^{Y}(\gamma)=\Lambda_{T}^{(Y)}(\gamma(0))=\exp \left[-\int_{0}^{T} \lambda_{Y}^{S}(\gamma(t)) d t\right],
$$

and the corresponding Lyapunov exponent of $\phi(\gamma)$ with respect to $X$ is

$$
L_{s}^{X}(\phi(\gamma))=\Lambda_{T}^{(X)}(\phi(\gamma(0)))=\exp \left[-\int_{0}^{T} \lambda_{X}^{s}(\phi \gamma(t)) d t\right] .
$$

This implies that the function $\eta(y)=\lambda_{Y}^{s}(y)-\lambda_{X}^{s}(\phi(y))$ has vanishing average over any periodic orbit of $Y$. Using the well known fact that $\phi$ is Hölder with a strictly positive exponent, we can conclude that $\eta$ is also Hölder. Since by assumption $Y$ was transitive, we can apply Livsic's theorem, [Li 1, Li 2], to conclude that there exists a Hölder function $\varrho: M \rightarrow \mathbb{R}$ such that

$$
\int_{0}^{t} \eta\left(\sigma_{s} y\right) d s=\varrho\left(\sigma_{t} y\right)-\varrho(y)
$$

for any $y \in M, t \in \mathbb{R}$. By (2), the last factor to estimate in (1) can be rewritten

$$
\begin{aligned}
& \left|\Lambda_{t}^{(X)}\left(\phi \circ \sigma_{-t} \circ \tilde{\phi}^{-1}(x)\right) / \Lambda_{t}^{(Y)}\left(\sigma_{-1} \circ \tilde{\phi}^{-1}(x)\right)\right| \\
& \quad=\exp \left[\varrho\left(\tau_{t} \circ \sigma_{-t} \circ \tilde{\phi}^{-1}(x)\right)-\varrho\left(\sigma_{-t} \circ \tilde{\phi}^{-1}(x)\right)\right] \leqq \exp \left(2\|\varrho\|_{C^{0}}\right) .
\end{aligned}
$$

This finishes the proof that $\phi$ is uniformly Lipschitz along stable manifolds of $Y$. Now we conclude that it is actually of class $C_{s}^{1}$.

By the fundamental theorem of calculus, the restriction of $\phi$ to any leaf of the stable foliation is differentiable almost everywhere on this leaf.

Also, since $\phi$ intertwines $\tau_{t}, \sigma_{t}$, the set of points where it is differentiable along the stable foliation is invariant under $\sigma_{t}$.

These two properties allow us to conclude that it is differentiable almost everywhere with respect to the forward Sinai-Ruelle-Bowen measure. 
At the points where $\phi$ is differentiable, we have that $\Lambda_{t}^{(X)}(\phi(y)) \xi(y)$ $=\xi\left(\sigma_{t}(y)\right) \Lambda_{t}^{(Y)}(y)$ or

$$
\frac{d}{d t} \ln \xi\left(\sigma_{t}(y)\right)=\lambda_{t}^{(X)}(\phi(y))-\lambda_{t}^{(Y)}(y)
$$

Since $S-R-B$ measure is ergodic, the solutions of the Livsic equation are unique in the class $L_{S-R-B}^{1}$.

Hence $\frac{d}{d t} \ln \xi$ has to agree a.e. $S-R-B$ with the Hölder function $\varrho$.

Using equation (3), we see that $\xi$ has to agree with $\varrho$ everywhere and it is standard that, therefore, it has to be the true derivative.

This proves $\phi$ is actually $C_{s}^{1}$.

One we know (3) holds everywhere we can very quickly bootstrap. Since $\lambda_{t} \in C_{s}^{\infty}$, assuming that $\phi \in C_{s(Y)}^{k}$ implies the right-hand side of (3) is in $C_{s(Y)}^{k}$. The proof of Lemma 2.2 in [LMM] allows us to conclude $\ln \xi$ is also in $C_{s(Y)}^{k}$, hence $\phi$ is in $C_{s(Y)}^{k+1}$.

This concludes the proof that $\phi \in C_{s(Y)}^{\infty}$. A very similar proof would conclude $\phi$ $\in C_{u(1)}^{\infty}$. Now we can apply Lemma 2.3 of [LMM] (another different proof was given by Journé [Jo] using different regularity assumptions, which also hold for the foliations considered here) to conclude $\phi \in C^{\infty}$ as claimed in Theorem 1.

Proof of the Lemma. We can assume that both $M$ and the foliations are orientable, since otherwise we could work on a suitable cover of $M$. Then we construct a global nowhere vanishing vector field $G$ of class $C_{\mathscr{G}}^{\infty}$, tangent to $\mathscr{G}$ everywhere, and another similar one, $F$ for $\mathscr{F}$. We consider a covering $U_{i}$ of $M$ by elementary open sets such that $\mathscr{G}$ is trivial on $V_{i}=\bigcup_{U_{J} \cap U_{i} \neq \emptyset} U_{j}$ for any $i, \sum_{i} \subset V_{i}$ is a transversal hypersurface to $\mathscr{G}$, and $\pi_{i}: V_{i} \rightarrow \sum_{i}$ is the projection along the leaves of $\mathscr{G}$.

We define $D_{j} \in C^{\infty}\left(V_{j}\right)$ for every $j$ by

$$
y=\exp _{D_{j}(y)}^{G} \pi_{j} y, \quad y \in V_{j},
$$

together with $D_{j}\left(\pi_{j} x\right)=0$. Roughly speaking, we are taking coordinates along the leaves in a coherent fashion for all the leaves. The reason why we do it using the exponential map is to ensure that the differential operators that result can be related for different leaves. Finally, we consider a $C^{\infty}$ partition of unity $\chi_{j}$ subordinated to $\left\{\phi^{-1}\left(U_{j}\right)\right\}$, and define

$$
\phi_{\varepsilon}(x)=\exp _{\left(S_{\varepsilon}-I\right)\left(\Sigma_{i} \chi_{2}(x) D_{\imath}(\phi(x))\right)}^{G} \phi(x), \quad x \in M,
$$

where $I$ is the identity operator acting on $C^{0}(M)$, and $S_{\varepsilon}$ is a $C_{\mathscr{F}}^{\infty}$-smoothing operator acting on $C^{0}(M)$ by

$$
S_{\varepsilon} \phi(x)=\int_{-\infty}^{\infty} \eta(t) \phi\left[\exp _{t \varepsilon}^{F}(x)\right] d t
$$

where $\eta \in C_{0}^{\infty}(\mathbb{R})$ is a nonnegative even function with $\int_{-\infty}^{\infty} \eta(t) d t=1$. The operator $S_{\varepsilon}$ can be seen in local coordinates for the leaves of $\mathscr{F}$ as a convolution operator, which approaches the identity as $\varepsilon$ tends to 0 . This implies that $\phi_{\varepsilon}$ tends to $\phi$ as $\varepsilon$ tends to 0 . 
It should be noted now that, even when we are working on a cover of $M$, the $\phi_{\varepsilon}$ above defines a map from $M$ to $M$. This is because $\eta$ is an even function. Moreover, it is obvious that any $\phi_{\varepsilon}$ sends each leaf of $\mathscr{F}$ to its image under $\phi$. In order to prove that each $\phi_{\varepsilon}$ is a homeomorphism for $\varepsilon$ near 0 , it suffices to see that they are injective. This can be seen easily as follows:

a) points in different leaves of $\mathscr{F}$ are mapped to different leaves of $\mathscr{G}$;

b) one-dimensional convolution operators transform strictly increasing functions into functions with strictly positive derivative, so the $\phi_{\varepsilon}$ 's are locally injective on each leaf, with uniform bounds on the size of the domain of injectivity.

Finally, we have to prove that each $\phi_{\varepsilon}$ is of class $C_{\mathscr{F}}^{\infty}$. This can be seen as follows: applying (4) to $\pi_{k} y$, assuming $y \in U_{j} \cup U_{k}$ and $U_{j} \cap U_{k} \neq \emptyset$, we have

$$
\pi_{k} y=\exp _{D_{j}\left(\pi_{k} y\right)}^{G} \pi_{j} y
$$

and using this with $j$ and $k$ interchanged in (4) again we have

$$
y=\exp _{D_{\jmath}(v)+D_{k}\left(\pi_{j} y\right)}^{G} \pi_{k} y .
$$

Comparing this with (4), we see that

$$
D_{k}(y)=D_{j}(y)+D_{k}\left(\pi_{j} y\right)
$$

As a consequence of this, assuming $y=\phi(x)$, we have for $x \in \phi^{-1}\left(U_{k}\right)$,

$$
D_{k}(\phi(x))=\sum_{j}\left[\chi_{j}(x) D_{j}(\phi(x))+\chi_{j}(x) D_{k}\left(\pi_{j} \phi(x)\right)\right]
$$

Using this in (4) as well as (5) and (6), we see that

$$
\phi_{\varepsilon}(x)=\exp _{S_{\varepsilon}\left(\Sigma_{i} \chi_{\imath}(x) D_{\imath}(\phi(x))+\Sigma_{i} \chi_{\imath}(x) D_{k}\left(\pi_{\imath} \phi(x)\right)\right.}^{G} \pi_{k} \phi(x) .
$$

Now it is clear that $\phi_{\varepsilon}$ is of class $C_{\mathscr{F}}^{\infty}$, since each $\pi_{j} \circ \phi$ is (locally) constant along the leaves of $\mathscr{F}, S_{\varepsilon}(\cdot)$ is of class $C^{\infty}$ by construction and $\exp _{t}^{G}(y)$ defines a continuous family of $C^{\infty}$ functions depending on the parameter $y$. This finishes the proof of the lemma.

Acknowledgements. The authors have benefited from hospitality at the Forschungsinstitut für Mathematik and Zentrum für Theoretische Physik at E.T.H. Zürich, where the first steps in the development of this work were given. The second author also thanks I.M.P.A., Rio de Janeiro, for a research stay in August and September 1986.

A grant from the Spanish-North American Committee for Scientific and Technological Cooperation to the second author made it possible for us to work together in Princeton, November 1986.

This work was partially supported by grant \#397/84 of the CAICYT.

\section{References}

[DG] Duistermaat, J., Guillemin, V.: The spectrum of positive operator and periodic bicharacteristics. Invent. Math. 29, 39-79 (1975)

[FO] Feldman, J., Ornstein, D.: Semi-rigidity of horocycle flows over compact surfaces of variable negative curvature. Ergodic Theory Dyn. Syst. 7, 49-73 (1987)

[Fr] Franks, J.: Anosov diffeomorphisms on tori. Trans. A.M.S. 145, 117-124 (1969)

[Jo] Journé, J.-L.: On a regularity problem occurring in connection with Anosov diffeomorphisms. Commun. Math. Phys. 106, 345-352 (1986) 
[Ka] Katok, A.: Entropy and closed geodesics. Ergodic Theory Dyn. Syst. 2, 339-364 (1982)

[Li 1] Livsic, A.: Homology properties of $Y$ systems. Math. Notes 10, 758-763 (1971)

[Li 2] Livsic, A.: Cohomology of dynamical systems. Math. U.S.S.R. Izv. 6, 1278-1301 (1972)

[L1] Llave, R. de la: Invariants for smooth conjugacy of hyperbolic dynamical systems. II. Commun. Math. Phys. 109, 369-378 (1987)

[LMM] Llave, R. de la, Marco, J.M., Moriyón, R.: Canonical perturbation theory of Anosov systems and regularity results for the Livsic cohomology equation. Ann. Math. 123, 537-611 (1986)

[MM] Marco, J.M., Moriyón, R.: Invariants for smooth conjugacy of hyperbolic dynamical systems. III. Commun. Math. Phys. 102, 317-333(1987)

[Ma] Manning, A.: There are no new Anosov diffeomorphisms on tori. Am. J. Math. 96, $422-429$ (1974)

[Ne] Newhouse, S.: On codimension one Anosov diffeomorphisms. Am. J. Math. 92, 761-770 (1970)

Communicated by J. N. Mather

Received April 17, 1987; in revised form October 22, 1987 